\title{
APROPIACIÓN DEL APRENDIZAJE DE HABILIDADES COORDINATIVAS EN NIÑOS SORDOS Y OYENTES
}

\section{APPROPRIATION OF THE LEARNING SKILL COORDINATIVAS IN DEAF CHILDREN AND LISTENERS.}

Huerta Ojeda, Álvaro Cristian; Fuchslocher Peñaloza, Karla Alejandra; Madrid Charme, Sebastián Ignacio \& Quiroz Barrientos, Alejandro Hugo.

Escuela Educación, Carrera de Educación Física - Universidad Santo Tomás, Sede Viña del Mar. Chile.

HUERTA O.A., FUCHSLOCHER P.K., MADRID Ch.S., QUIROZ B.A. Apropiación del aprendizaje de Habilidades Coordinativas en niños sordos y oyentes. Mot. Hum. 14(2): 46-51.

\section{RESUMEN}

Objetivo: Determinar el grado de asimilación de habilidades coordinativas en niños sordos de edades entre seis a ocho años, en relación con niños oyentes de la misma edad. Materiales y Método: La investigación tiene un enfoque cuantitativo con un alcance de tipo correlacional y un diseño metodológico Cuasi Experimental - Causal Comparativo. La muestra corresponde a 20 alumnos (Experimental $1 n=10$ - sordos y Experimental $2 n=10$-oyentes) elegidos de manera no probabilística intencionada. La ejecución del instrumento de medición contempla un Test y un Post Test con pruebas enfocadas a medir la Habilidades Coordinativas Generales (H.C.G.). Cada ejecución contempla una duración de una hora y treinta para ambos grupos. Para el análisis de datos se utilizaron la t- Student y ANOVA de una vía. Análisis e Interpretación de datos: El análisis para las H.C.G. entre ambos grupos entre el Test y Post Test, arrojó un p valor = 0,0023, lo que indica que existe una diferencia muy significativa en la capacidad mencionada. Conclusiones: Al término de la investigación, los niños sordos presentaron mayor grado de asimilación en las H.C.G. al compararlos con los niños oyentes de la misma edad ( $p=0,0023)$, lo que avala la hipótesis de investigación.

Palabras Clave: Aprendizaje motor, habilidades motoras básica, coordinación y sordera.

\begin{abstract}
Objective: To determine the degree of assimilation of coordinative skills in children who are deaf and their age is between six to eight years old, in connection with children who can hear of the same age. Materials and Methods: The research has a quantitative approach with a range of a correlational type and quasi-experimental study design causativecomparative. The sample corresponds to 20 students (One Experimental $=10$ - Deaf and Two Experimental $=10$ listeners) selected on a non-probabilistic intentional. The execution of the measurement instrument includes a test and post-test with tests focused on measuring general coordinative abilities (H.C.G.). Each performance includes a duration of one hour and thirty minutes for both groups. For data analysis, we used the T student and a one-way ANOVA. Analysis and interpretation of data: H.C.G. analysis for both groups between the Test and Post Test yielded a value of 0.0023, indicating that there is a very significant difference in the listed capacity. Conclusions: At the end of the investigation, deaf children had a higher degree of assimilation into the HCG when compared to the hearing children of the same age ( $p$ $=0.0023)$, which supports the research hypothesis.
\end{abstract}

Key Words: Motor learning basic, motor skills, coordination and deaf. 


\section{INTRODUCCIÓN.}

El ser humano siempre ha tenido la necesidad imperante de moverse, de descubrir y aprender a través del movimiento, desarrollando diferentes habilidades y capacidades físicas que posteriormente sentarán la base para un mejor desarrollo y resolución de problemas. Pero esto no sería posible sin la ayuda de los sentidos, responsables directos de captar el mundo externo en relación a nuestro cuerpo. Éstos empiezan a formarse desde la gestación; a la cuarta semana se desarrolla el sentido de la vista, a la sexta semana sigue el desarrollo del gusto y el olfato, a la séptima semana el tacto y por último, a la décima sexta semana, se da lugar al sentido de la audición.

Todo movimiento requiere coordinación y equilibrio, es en este punto en que radica la importancia del desarrollo, aprendizaje y asimilación de la ejecución motriz a temprana edad. Obtener una buena coordinación producto de una pronta estimulación, repercutirá beneficiosamente en la vida cotidiana del niño, desarrollando "la capacidad neuromuscular de ajustar con precisión lo requerido y pensado de acuerdo con la imagen fijada mediante la visión e inteligencia motriz a la necesidad del movimiento" (Contreras, 1998).

Los sentidos permiten percibir señales, reflejos, propiedades y atributos del mundo exterior y de la propia corporeidad. Se menciona que "el oído interno contiene el órgano del equilibrio (conducto semicirculares de los cuales se obtiene información sobre la posición de la cabeza respecto de la horizontalidad). Esto explica que algunas enfermedades que causan sordera también afectan el sentido del equilibrio y pueden causar vértigos y mareos. Aparte de estas consecuencias de tipo perceptivo, encontramos también las motoras, entre las que están el retraso en la motricidad fina, deficiente coordinación general del cuerpo en carreras y saltos, problemas en orientación espaciotemporal, desarrollo de la lateralidad, y retraso en la adquisición del esquema corporal correcto" (Sáez y Monroy, 2008).

Autores corroboran que "el oído interno está formado por un conjunto de cavidades labradas en el interior del peñasco, denominadas laberinto. Se encuentran íntimamente relacionadas la porción correspondiente al equilibrio (sistema vestibular) y a la audición (sistema coclear)" (Ramírez, 1982). Otros, mencionan que ante la falta de los sentidos dominantes, existe una utilización relativa de los restantes con el objetivo de compensar dicha carencia, lo que influye directamente en su esquema de conducta, la que se desenvuelve de forma diferente a la del niño oyente; la capacidad de respuesta del niño sordo ha de sufrir una serie de procesos cuya superación lo capacitan para obtenerla (Marchesi, 1987).

Marchesi (1987), señala que el sordo, al carecer de la vía sensorial auditiva, realiza en gran medida toda su exploración y comprensión del mundo mediante otras vías compensatorias (primordialmente la visual y kinestésica), esto de alguna manera podría explicar que en algunos casos existiría la posibilidad que las personas sordas en comparación con sus pares oyentes, sean más perceptivas en estas dos áreas. Se sabe además que las personas sordas son capaces de ocupar en su totalidad el campo visual, procesar más rápidamente las percepciones visuales y establecer diferentes asociaciones.

Según Muñoz (Muñoz, 2009), "un correcto equilibrio es la base fundamental de una buena coordinación dinámica general y de cualquier actividad autónoma", pero sabemos que la sordera afecta el equilibrio, y por lo tanto el niño sordo deberá compensar esta deficiencia a través de otras vías tales como: sistema visual y sistema kinestésico. Además hay que considerar, que producto de esta compensación sensorial, las personas sordas podrían percibir y asimilar de mejor manera cualquier tipo de información motora entregada.

Es probable que surjan problemas en el buen desarrollo de estos sentidos desde la más temprana edad, ya que el niño está expuesto y vulnerable a diferentes agentes patógenos tanto internos como externos, que pueden perturbar en diferentes grados la funcionalidad de los sentidos del organismo, acarreando problemas de carácter permanente como la sordera (Heward, 1998). 
$\begin{array}{ccr}\text { La encuesta } & \text { de } & \text { Caracterización } \\ \text { Socioeconómica } & \text { Nacional } & \text { (CASEN) }\end{array}$ correspondiente al año 2006, revela que de un total de "1.119.867 discapacitados en Chile, 197.739 corresponden a sordos o personas con discapacidad auditiva incluyendo a quienes ocupan audífonos" (MIDEPLAN, 2006).

Algunos autores señalan que "cuando los sistemas vestibular, propioceptivo y táctil no trabajan bien, el niño esta propenso a tener una coordinación insuficiente. Puede perder el equilibrio y tropezar fácilmente" (Ayres, 1998). En algunos casos la sordera influye en las habilidades motrices. Al estar dañado el oído interno, específicamente en los anillos semicirculares se tiene problemas con el equilibrio, el cual afectará otras áreas como la ubicación témporo espacial, lateralidad, coordinación dinámica general, etc. Esta última cualidad motriz (coordinación dinámica general), es la encargada de ayudar al individuo a realizar acciones cinéticas precisas y equilibradas y reacciones rápidas adaptadas a cada situación.

Considerando que algunos sordos no cuentan con el sistema auditivo funcional, producto de alteraciones del oído interno, tienen la necesidad de descubrir y vislumbrar el mundo ocupando sólo los restantes sentidos. Pero, si estas personas son reforzadas de manera automática en aspectos visuales y kinestésicos ¿podrán compensar y suplir las deficiencias en el sistema auditivo? Acá, tanto la visión como el sistema kinestésicos se podrían volver más perceptivos y suplir la carencia del sistema macular (que se encarga parcialmente de enviar señales a centros integradores para ayudar a mantener el equilibrio), pudiendo ser capaces de asimilar aspectos motrices de forma más facilitada.

Por todo lo anterior, la presente investigación pretende comparar la apropiación del aprendizaje de Habilidades Coordinativas en niños sordos del Centro de Estudios y Capacitación para Sordos (CECASOV) y niños con audición normal del Colegio Juanita Fernández, de Viña del Mar, Chile, de edades entre seis y ocho años.

\section{MATERIAL Y MÉTODO.}

La investigación posee un alcance correlacional, con un diseño Cuasi Experimental Causal Comparativo, contemplando un Test y un Post Test para ambos Grupos Experimentales. Además, se utilizó un evaluado externo a los investigadores (un ciego), despejando los sesgo entre los sujetos sordos y oyentes (Hernández, 2006).

\begin{tabular}{|c|c|c|}
\hline & $\begin{array}{l}\mathbf{X}_{1.1} \\
\mathbf{X}_{2.1} \\
\end{array}$ & $\begin{array}{l}\longrightarrow \\
\longrightarrow \\
\longrightarrow\end{array}$ \\
\hline $\begin{array}{l}\mathrm{X}_{1.1} \\
\mathrm{X}_{1.2}\end{array}$ & $\begin{array}{l}=\text { Medición inicial } \mathrm{GE}_{1} . \\
=\text { Medición final } \mathrm{GE}_{1} .\end{array}$ & $\begin{array}{ll}\mathrm{X}_{2.1 .} & =\text { Medición inicial } \mathrm{GE}_{2} . \\
\mathrm{X}_{2.2 .} & =\text { Medición final } \mathrm{GE}_{2} .\end{array}$ \\
\hline
\end{tabular}

Figura 1. Diseño de Investigación con ambos grupos.

Sujetos.

De carácter no probabilística intencional, conformada por un total de 20 sujetos, divididos en dos grupos de 10 alumnos cada uno. El primero denominado Grupo Experimental 1, compuesto por sordos edades entre 6 y 8 años, muestra correspondiente al 100 por ciento de la población y al 19,23 por ciento del universo del Colegio
CECASOV de Valparaíso. El segundo denominado Grupo Experimental 2, constituido por 10 alumnos oyentes de edades entre 6 y 8 años, grupo que corresponde al 41,66 por ciento de la población y al 2,4 por ciento del universo del Colegio Juanita Fernández ubicado en Viña del Mar. 


\section{Selección del Instrumento de Evaluación.}

El instrumento seleccionado para esta investigación, fue el Test "Batería para Evaluar Rendimiento Motor y Coordinación (BAPERMOC)", en el cuál uno de sus subtipos nivel básico (B-1-1) está destinado a medir factores de la coordinación. Éste fue extraído de la Batería de Pruebas creada por el Centro de Perfeccionamiento, Experimentación Investigaciones Pedagógicas (CPEIP),

\section{RESULTADOS}

Tabla 1. Comparación de H.C.G. entre el Test y Post Test Para del GE 1 y GE 2 .

\begin{tabular}{ccccc}
\hline GRUPOS & Test sordos & $\begin{array}{c}\text { Post Test } \\
\text { sordos }\end{array}$ & Test oyentes & $\begin{array}{c}\text { Post Test } \\
\text { oyentes }\end{array}$ \\
\hline MEDIA & 8,9 & 10,1 & 7,2 & 8,0 \\
\hline DS & 1,28 & 1,52 & 1,87 & 1,76 \\
\hline
\end{tabular}

En la tabla $\mathrm{N}^{\mathrm{o}} 1$, se aprecian las medias de los dos grupos en Test y Post Test, siendo las mediciones iniciales $8,9 \pm 1,28$ y $7,2 \pm 1,87$ puntos para $\mathrm{GE}_{1}$ y $\mathrm{GE}_{2}$ respectivamente. Además, en el Post Test en ambos grupos, se observan valores de 10,1 $\pm 1,52$ y $8,0 \pm 1,76$ puntos para $\mathrm{GE}_{1}$ y $\mathrm{GE}_{2}$ respectivamente.
Asimismo, el $\mathrm{p}$ valor calculado $(\mathrm{p}$ valor $=0,0023)$ permite inferir que entre los datos existen diferencias Muy Significativas en las H.C.G. entre ambos grupos.

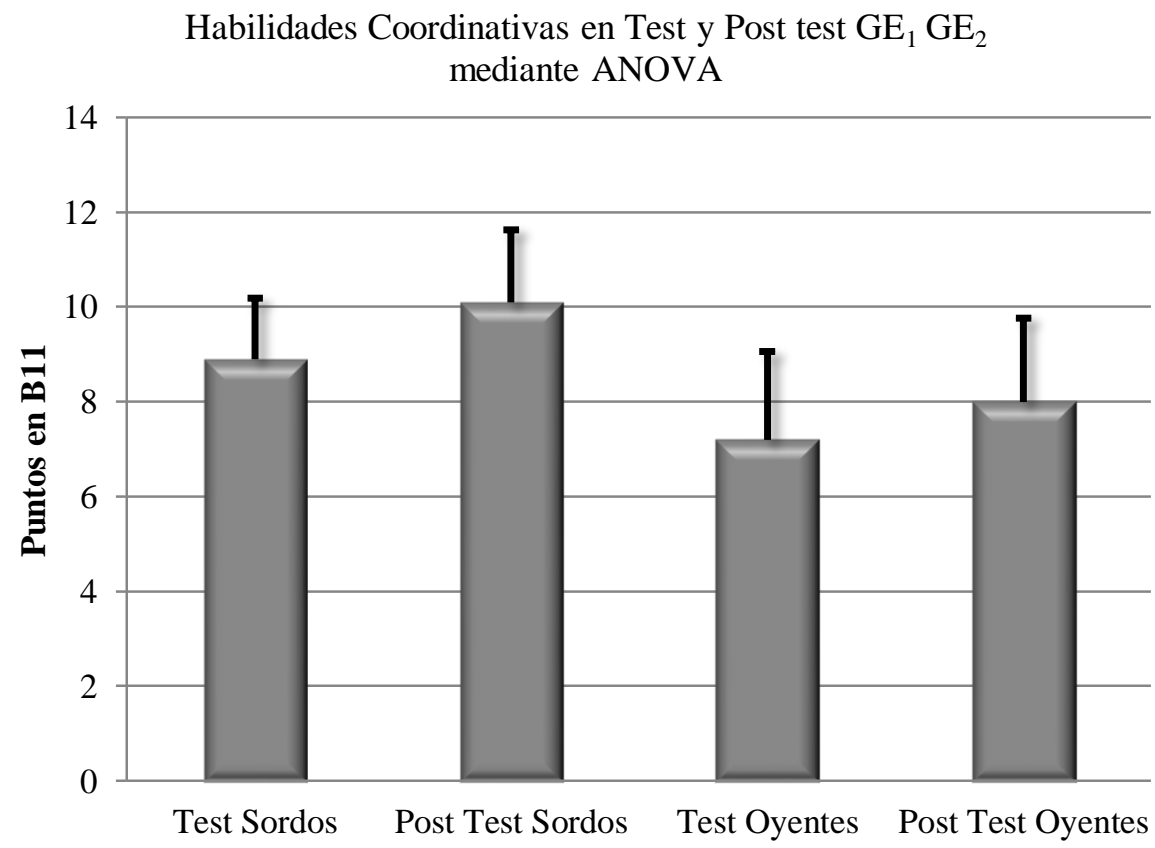

Figura 2. Comparación puntaje en H.C.G. en Test y Post Test para el GE y GE$_{2}$.

En la figura $\mathrm{N}^{\circ} 2$, se aprecian las diferencias de ambas muestras entre el Test y el Post Test. Dicha diferencia, según el $\mathrm{p}$ valor calculado $(\mathrm{p}=0,0023)$, representa una diferencia muy significativa en la variable de H.C.G. entre $\mathrm{GE}_{1}$ y $\mathrm{GE}_{2}$. 


\section{DISCUSIÓN.}

La niñez temprana (2 a 7 años de edad), es una etapa fundamental para el desarrollo armónico y balanceado del ámbito cognoscitivo, afectivo y psicomotriz del comportamiento humano (Mcclenaghan, 1985). El apto desarrollo de estas H.M.B, será decisivo para formar una base más sólida para futuras experiencias y actividades motrices más complejas y específicas como las deportivas, entregando a su vez herramientas a la persona para su desenvolvimiento en las actividades del diario vivir.

Hay autores que mencionan que la niñez es la "cuna de oro" para los aprendizajes "entendiendo que esta edad de oro, no llegará con garantías de explotar toda su potencialidad, si no se ha realizado un trabajo programado desde la base; es decir, las edades comprendidas entre 0 y 6 años" (Beraldo \& Polleti, 1991). El trabajo de la capacidad coordinativa debería ser el cimiento sobre el que se construya el repertorio gestual de cualquier niño. También, se afirma que entre los 4 y 7 años "es cuando las mejoras coordinativas experimentan su mejor grado de crecimiento" (Hahn, 1998.); Beraldo y Polleti (1991), anuncian que "la mejor edad para intervenir sobre los procesos coordinativos, va desde los 6 a 11 años".

La infancia es un periodo de la vida en la cual van madurando las facultades relacionadas con el desarrollo del lenguaje en el niño, siendo estas la agudeza auditiva y visual, la coordinación sensoriomotriz, la motricidad fina y gruesa, capacidades de carácter mental tales como la memoria, atención, comunicación y otras que van floreciendo con los años, asentando base para el aprendizaje escolar.

Patologías como de la sordera, pueden generar problemas en los individuos a nivel perceptivo motor, ya que "al no tener control auditivo existe una torpeza motora y un andar pesado y poco seguro que hacen, en muchos casos, que el niño con sordera vaya superando esas fases con un ligero retraso respecto al oyente" (Sáez \& Monroy, 2008). También se señala que "cuando los sistemas vestibular, propioceptivo o táctil no trabajan bien, el niño esta propenso a tener una coordinación insuficiente. Puede perder el equilibrio y tropezar fácilmente" (Ayres, 1998), sobre todo si hay problemas en el sistema vestibular, ya que "el oído interno está formado por un conjunto de cavidades labradas en el interior del peñasco, denominada Laberinto. Se encuentran íntimamente relacionadas la porción correspondiente al equilibrio" (Ramírez, 1982).

Pero, "ante la carencia de los sentidos predominantes, existe una transposición de la utilización relativa de los restantes, lo que influye en su esquema de conducta, que se desenvuelve de forma diferente a la del niños oyente; la capacidad del niño sordo sufre una serie de procesos cuya superación lo capacitan para obtenerla" (Ramírez, 1982). Es más, si existe la posibilidad de divisar a un niño sordo cuando está jugando, se puede apreciar que voltea constantemente su cabeza para mirar lo que ocurre a su alrededor percibiendo el mundo externo con su vista, junto con la ayuda del tacto y las vibraciones (Flores \& Barruecos, 2006). Esto se debe a que "el oído capta estímulos en un fondo envolvente, pero cuando no existe la vista debe convertirse en el sentido "líder", ya no sólo para lo que el sujeto tiene enfrente, sino también para lo que está detrás de él”.

En casos de perdidas auditivas graves, el niño genera una "exploración y comprensión del mundo utilizando vías compensatorias" (Marchesi, 1987). Refiriéndose a los sentidos restantes los cuales tratarán de regular el déficit que ha dejado el sentido dañado, todo esto porque el sordo "se muestra psicológicamente más sensible a ciertas partes del cuerpo humano relacionadas con los sentidos que más utiliza (visión, tacto, gusto y olfato)" (Ramírez, 1982).

\section{CONCLUSIONES.}

De acuerdo a los resultados obtenidos en esta investigación, podemos señalar que la hipótesis se avala. No obstante, las conductas de entrada en ambos grupos no fueron iguales $(\mathrm{p}=0,0001)$; este dato fue obtenido a través del método t-Student, acá se señala que entre $\mathrm{GE}_{1}$ y $\mathrm{GE}_{2}$, al comienzo del tratamiento existió una diferencia Externadamente Significativa en la H.M.B. 
También se concluye que la sordera no es impedimento para realizar actividades motrices, ya que las personas sordas al carecer de su sentido auditivo, compensan y suplen esta vía con otras tales como el sentido visual y kinestésico, ambas vías son esenciales para un apto aprendizaje y desarrollo de conductas motrices relacionadas con el equilibrio y coordinación. Se debe considerar a las personas que presentan esta diversidad funcional (pérdida auditiva), como sujetos normales, capaces de desarrollar las habilidades coordinativas generales, de igual manera que una persona que no presenta este trastorno.

\section{REFERENCIAS BIBLIOGRÁFICAS}

Heward, W. (1998). Niños Excepcionales: Una introducción a Educación Especial. Editorial Prentice Hall. Madrid, España. 95,438 .

MIDEPLAN. (2006). Encuesta CASEN 2006: Discapacidad, (en línea). Chile: disponible en: http://www.mideplan.cl.

Ayres J. (1998). La integración sensorial y el niño. Editorial Trillas. Trillas, México. 77.

Contreras O. (1998). Didáctica de la Educación Física. Un enfoque constructivista. Editorial Inde. Barcelona, España. 189.

Saez G.; Monroy, A. (2008). Discapacidad y Educación Física: La Sordera. IV Congreso Internacional y XXV Nacional de Educación Física. Universidad de Córdoba, España. 2-5 Abril de 2008.

Ramirez R. (1982). Conocer al niño Sordo. Editorial CEPE. Madrid, España. 40, 47.

Marchesi A. (1987). El desarrollo cognitivo y lingüístico de los niños Sordos. Editorial Alianza. Madrid, España. 214.

Muñoz D. (2009). Revista digital Efdeportes. La coordinación y el equilibrio en el área de la Educación Física. Actividades para su desarrollo (en línea). . Disponible en: http//www.efdeportes.com/efd130/la-coordinacion-y-elequilibrio-en-el-area-de-educacion-fisica.htm.

Hernández R. (2006). Metodología de la Investigación. Editorial McGraw-Hill/Interamericana Editores S.A. D.F., México. 41, 44, 46, 49, 119, 238-242.

Mcclenaghan, B. (1985). Movimientos Fundamentales. Editorial Panamericana. Buenos Aires, Argentina. 1985:21,15.

Beraldo, S.; Polleti, C. (1991). Preparación física total. Editorial Hispano Europea. Barcelona, España. 57, 88.
Hahn E. (1998). Entrenamiento con niños. Editorial Martínez Roca, Barcelona, España.

Saez G.; Monroy A. (2008). Discapacidad y Educación Física: La sordera. IV Congreso Internacional y XXV Nacional de Educación Física, 2-5 Abril. Universidad de Córdoba, España.

Flores L.; Barruecos P. (2006). Los problemas de audición en la edad escolar. Editorial Trillas. Trillas, México. 25.

\section{Dirigir Correspondencia a:}

Álvaro Huerta Ojeda

Av. Limonares \# 190, Viña del Mar - Quinta Región - CHILE e-mail: achuertao@yahoo.es

RECIBIDO: 19-05-2013

ACEPTADO: 1-12-2013 